\title{
Arterial intracranial thrombosis as the first manifestation of vaccine-induced immune thrombotic thrombocytopenia (VITT): a case report
}

\author{
Michelangelo Mancuso ${ }^{1}$ (D) - Dario Luca Lauretti ${ }^{2} \cdot$ Nadia Cecconi $^{3} \cdot$ Massimo Santini $^{4} \cdot$ Valentina Lami $^{4}$. \\ Giovanni Orlandi ${ }^{1}$. Sergio Casagli ${ }^{5} \cdot$ Antonella Ghetta $^{5} \cdot$ Gaetano Liberti $^{6} \cdot$ Benini Maria Elena $^{6} \cdot$ Gabriele Siciliano $^{1}$. \\ Mirco Cosottini ${ }^{2}$
}

Received: 21 October 2021 / Accepted: 2 December 2021 / Published online: 13 January 2022

(c) Fondazione Società Italiana di Neurologia 2021

\begin{abstract}
Objective We describe a severe case of vaccine-induced immune thrombotic thrombocytopenia (VITT) after the first dose of the ChAdOx1 nCoV-19 vaccine leading to massive ischemic stroke.

Methods A 42-year-old woman developed acute left hemiparesis (NIHSS 12) 9 days after the first vaccine dose.

Results The blood tests revealed low platelets $\left(7010^{3} / \mu \mathrm{L}\right)$ and severe increment of D-dimer $(70,745 \mathrm{ng} / \mathrm{mL}$ FEU). Brain non-contrast computed tomography and multiphasic CT angiography demonstrated a right middle cerebral artery occlusion. The patient was treated with primary thrombectomy, steroids, immunoglobulin, and fondaparinux. Despite the treatment, the neurological status deteriorated and underwent decompressive hemicraniectomy. She was transferred to the rehab's unit 52 days after the onset.

Discussion Healthcare providers should be aware of the possibility of ischemic stroke as a manifestation of VITT. Awareness on this very rare and possibly fatal complication should be reinforced on both the vaccine recipients and general practitioners.
\end{abstract}

Keywords VITT $\cdot$ Vaccine $\cdot$ COVID-19 $\cdot$ Stroke $\cdot$ Thrombocytopenia

\section{Introduction}

Michelangelo Mancuso and Dario Luca Lauretti contributed equally to this work

Michelangelo Mancuso

michelangelo.mancuso@unipi.it

1 Department of Clinical and Experimental Medicine, Neurological Clinic, University of Pisa, Pisa, Italy

2 Department of Translational Research and New Technologies in Medicine and Surgery, University of Pisa, Pisa, Italy

3 Clinical and Experimental Medicine, University of Pisa, Pisa, Italy

4 U.O. Medicina D’Urgenza E Pronto Soccorso, Emergency Department, Nuovo Santa Chiara Hospital, Azienda Ospedaliero-Universitaria of Pisa, Pisa, Italy

5 Neuroanestesia E Rianimazione, Nuovo Santa Chiara Hospital, Azienda Ospedaliero-Universitaria of Pisa, Pisa, Italy

6 Neurosurgery Unit, Azienda Ospedaliero-Universitaria of Pisa, Pisa, Italy
Inactivated or live-attenuated viruses as well as recombinant proteins and vector technologies have been deployed to develop the COVID-19 vaccine. In addition, new platforms such as RNA and DNA vaccines are also used for the first time in a licensed vaccine [1]. Vaccine-induced immune mediated thrombotic thrombocytopenia (VITT) is a rare phenomenon of thrombosis with thrombocytopenia, sometimes associated with cerebral venous thromboses, now described following vaccination with adenovirus vaccines ChAdOx1 nCOV-19 (Oxford/AstraZeneca, AZ) and Ad26. COV2.S Johnson and Johnson (Janssen/J\&J) [2]. The evaluation and management of such events are challenging for the healthcare providers. Isolated ischemic stroke is very rarely observed. Here, we describe of a juvenile severe ischemic stroke and no evidence of venous thromboses following $\mathrm{AZ}$ vaccine. 


\section{Case report}

A 42-year-old Italian woman was admitted to our emergency unit for acute onset of left hemiparesis. Nine days prior the presentation, she received the first dose of the AZ vaccine. Her past medical history was negative. On examination, she was conscious and confused (Glasgow Coma Scale (GCS) $15 \backslash 15$ and NIHSS 12). At admission, D-dimer was severely increased $(70,745 \mathrm{ng} / \mathrm{mL}$ FEU, n.v. $<500)$, platelet count $7010^{3} / \mu \mathrm{L}$ (n.v. 140-450), and fibrinogen 214 mgldl (n.v. 200-400) (Supplementary Table). Her molecular COVID-19 test was negative. Brain non-contrast computed tomography, multiphasic $\mathrm{CT}$ angiography (CTA), and CT perfusion (CTP) showed early signs of ischemia on the right middle temporal gyrus, insula and putamen (ASPECT score 7), occlusion of the right middle cerebral artery (MCA), and a large area of penumbra (Fig. 1). The patient was immediately transferred to the angio suite and underwent general anesthesia for the procedure. The digital subtraction angiography (DSA) confirmed the MCA occlusion and revealed a further anterior cerebral artery (ACA) occlusion. Endovascular thrombectomy was performed by direct aspiration on the MCA and by combined technique using retrieval stent and distal aspiration on the ACA, obtaining a complete arterial recanalization (Fig. 2a, b). However, the control angiograms showed further multiple sites of intra-arterial aggregation involving to the right ophthalmic artery, (Fig. 2c) and the ACA that was re-occluded. (Fig. 2d). The pathological analysis of the thrombus revealed FVIIIRAgpositive platelets. Suspecting VITT, after taking samples for confirmation tests (PF4-ELISA test and HIPA assay), therapy was immediately started, with dexamethasone (40 mg i.v. for 4 days), i.v. Ig-HD ( $1 \mathrm{~g} / \mathrm{Kg}$ for 4 days), s.c. fondaparinux (5 $\mathrm{mg}$ the first day and then different posology depending on platelet count), and platelet infusion at day 4 and 7 after the stroke onset [3] (Fig. 3). The HIPA assay and the anti-PF4 ELISA were positive, confirming the suspicion of VITT.

One day later, the patient deteriorated. A repeat brain CT showed a large hemorrhagic transformation within the ischemic region, and she underwent decompressive hemicraniectomy. Ultrasound screening of venous thrombosis at the neck, upper and lower arms, at the portal, splanchnic, or hepatic veins was negative. Transthoracic echocardiography was normal, and right-to-left shunt was excluded with transcranial Doppler. Assessment for inherited (protein $C$ and $\mathrm{S}$ and anti-thrombin III deficiency, V Leiden mutation, and prothrombin G20210A mutation) and acquired (antiphospholipid syndrome and thrombotic thrombocytopenic purpura) thrombophilia was negative.

She was discharged after 52 days and transferred to the severe brain injury rehabilitation unit. After almost 5 months from the stroke, she is fully oriented, walks with aid, has a moderate paresis of the left arms, lost the vision in the right eye, and can normally talk and swallow.

The patient's legal representative provided written informed consent to publish the case.

\section{Discussion}

We report a very rare $(<1 / 100,000$ doses administered, as defined by the AIFA, https://www.aifa.gov.it/docum ents/20142/1315190/Rapporto_sorveglianza_vaccini_
Fig. 1 Non-contrast computed tomography at basal ganglia (a) and lateral ventricles level (a1) showed early signs of ischemia (loss of gray-white matter differentiation) involving the right middle temporal gyrus, insula, and putamen. (b) Multiphasic CT angiography demonstrates the right MCA occlusion (arrow) with good collateral circulation (b1). (c) CT perfusion maps: the reduction of cerebral blood volume (CBV) confirms the areas of irreversibly infarcted tissue; the largest area of increased the mean transit time (MTT) (c1) borders the penumbra area as the mismatch with CBV map
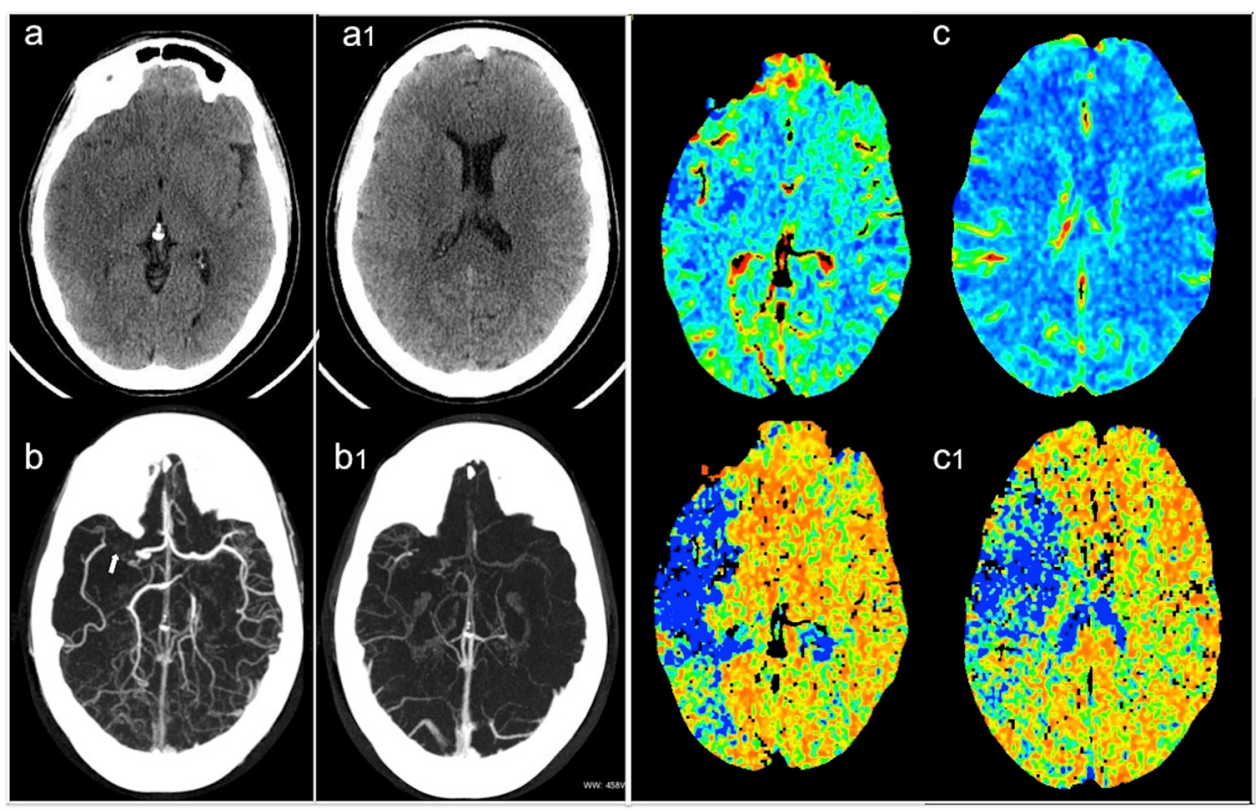


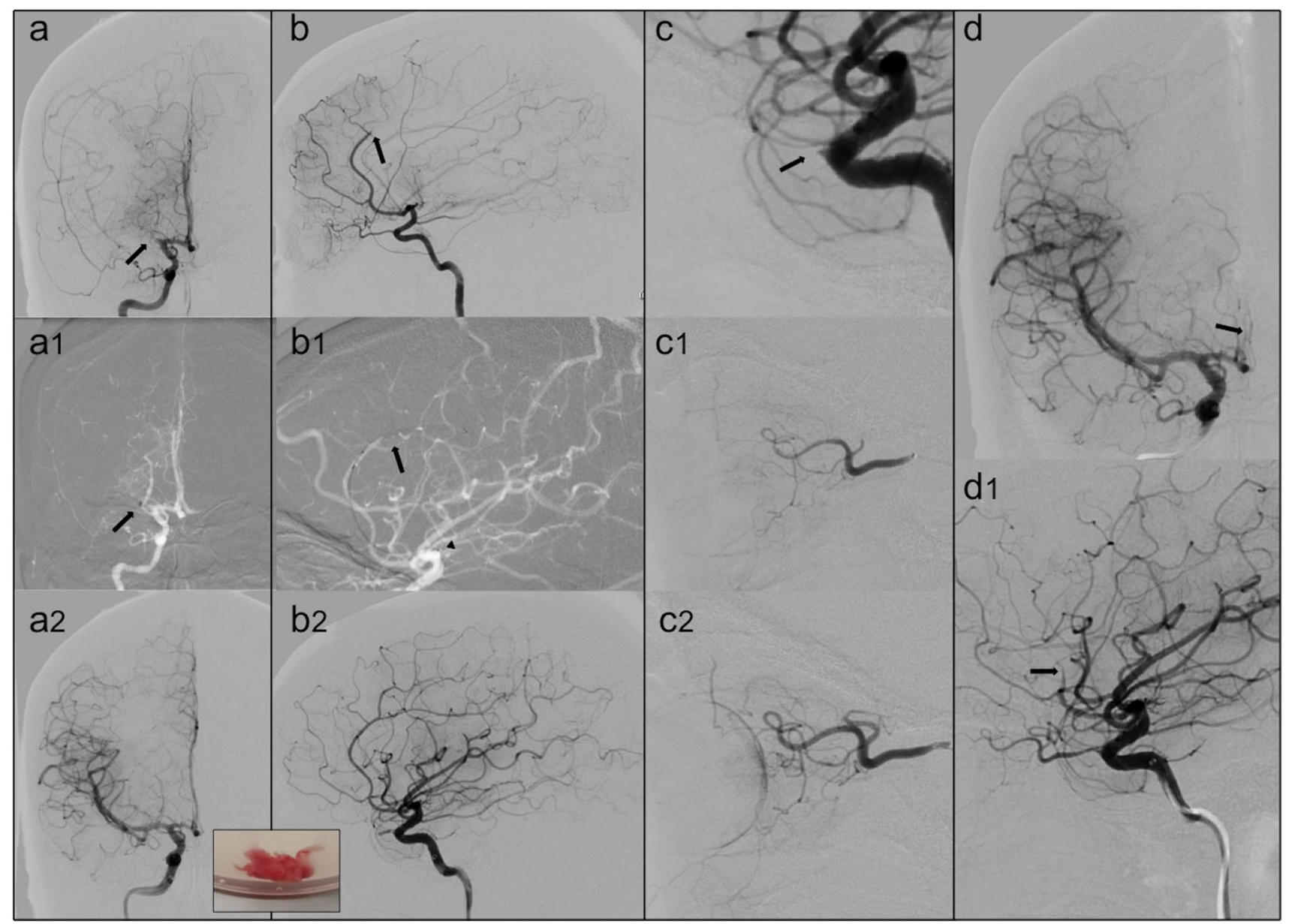

Fig. 2 (a) The digital subtraction angiography (DSA) in frontal view confirms the occlusion of the right MCA (arrow). During the endovascular procedure (a1), the distal tip of the aspiration catheter (arrow) engages the thrombus with complete MCA recanalization (a2). In the box, the macroscopic aspect of the removed clot is shown. (b) DSA in lateral view reveals the occlusion of the A3 segment of the anterior cerebral artery (arrow). A combined technique with a stent retriever positioning thought thrombus in the pericallosal artery (arrow) and distal tip of aspiration catheter (arrowhead) at the origin of the vessel (b1) allows the ACA recanalization (b2). (c) DSA in lateral view: after the recanalization of intracranial vessels a right ophthalmic artery occlusion appeared (arrow). Micro-catheterization with intra-arterial alteplase infusion was performed (c1) with flow restoration (c2). (d) DSA in frontal and lateral view demonstrates the formation of further thrombi (arrows) with re-occlusion of the ACA

leading to reduced platelet count and results in thrombosis, similar to heparin-induced thrombocytopenia [4]. Treatment of this syndrome is challenging; very recently, the Italian Society for the Study of Haemostasis and Thrombosis has proposed therapeutic options [3] that in our patient lead to a good response on platelets count (Fig. 3).

The clinical spectrum of coagulopathy associated with COVID-19 vaccination is its broad. In the past few months, several cases of VITT following AZ vaccination have been reported. Most of the patients included in these reports were women younger than 50 years of age and had thromboses at unusual sites, including cerebral venous sinus thrombosis, thrombosis in the portal, splanchnic, or hepatic veins, deep venous thrombi [5-10]. Very recently, two cases with severe ischemic stroke and pulmonary and portal vein thrombosis 


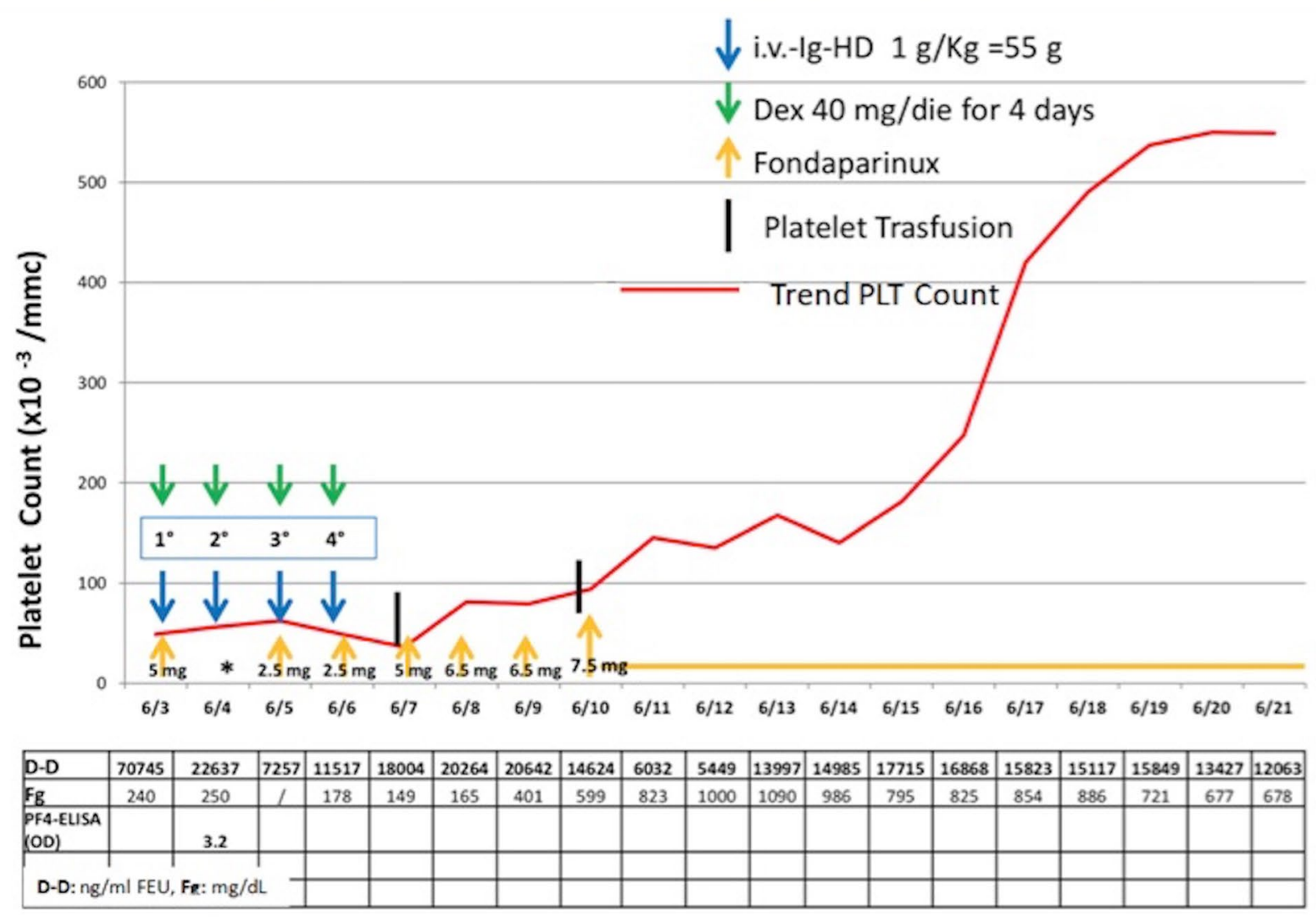

Platelet Count Responses to Treatment: ${ }^{*}$ indicates the time at which the results of platelet factor 4 (PF4)-polyanion antibody tests were known

Fig. 3 Platelet count response to treatments. Dex, dexamethasone; i.v.-Ig, intravenous immunoglobulins; D-D, d-dimer; Fg, fibrinogen

have been reported [11], as well as one more case with occlusion of MCA and normal platelet count [12], one case of occlusion of MCA and low platelets [13], and multiple sites of arterial thrombosis requiring femoral and carotid surgical thrombectomy [14].

Our case is a very rare report of severe arterial thrombosis without evidence of venous involvement. The unusual behavior of multisegmental and recurrent arterial occlusions despite the endovascular thrombectomy could suggest an underlying systemic thrombogenic condition in patient who underwent to AZ vaccination. Healthcare providers should be aware of the possibility of such very rare and possibly fatal complication. Awareness should be reinforced on both the vaccine recipients and physicians; early identification of patients at their first clinical manifestation helps undertake all actions to reduce or avoid the dramatic consequences of VITT.

Supplementary Information The online version contains supplementary material available at https://doi.org/10.1007/s10072-021-05800-3.

\section{Declarations}

Ethical approval The authors report no disclosures relevant to the manuscript.

Conflict of interest The authors declare no competing interests.

\section{References}

1. Li YD, Chi WY, Su JH, Ferrall L, Hung CF, Wu TC (2020) Coronavirus vaccine development: from SARS and MERS to COVID19. J Biomed Sci 27.https://doi.org/10.1186/S12929-020-00695-2

2. Klok FA, Pai M, Huisman MV, Makris M (2021) Vaccine-induced immune thrombotic thrombocytopenia. Lancet Haematol 165. https://doi.org/10.1016/S2352-3026(21)00306-9

3. Gresele P, Marietta M, Ageno W et al (2021) Management of cerebral and splanchnic vein thrombosis associated with thrombocytopenia in subjects previously vaccinated with Vaxzevria (AstraZeneca): a position statement from the Italian Society for the Study of Haemostasis and Thrombosis (SISET). Blood Transfus. https://doi.org/10.2450/2021.0117-21 
4. Cines DB, Bussel JB (2021) SARS-CoV-2 vaccine-induced immune thrombotic thrombocytopenia. N Engl J Med 384:22542256. https://doi.org/10.1056/NEJME2106315

5. Schulz JB, Berlit P, Diener HC et al (2021) COVID-19 vaccineassociated cerebral venous thrombosis in Germany. Ann Neurol. https://doi.org/10.1002/ANA.26172

6. Bano F, Badugama B, Chandra D (2021) Thrombosis and thrombocytopaenia after ChAdOx $1 \mathrm{nCoV}-19$ vaccination: a single UK centre experience. BMJ Case Rep 14. https://doi.org/10.1136/ BCR-2021-243894

7. Tølbøll Sørensen AL, Rolland M, Hartmann J et al (2021) A case of thrombocytopenia and multiple thromboses after vaccination with ChAdOx1 nCoV-19 against SARS-CoV-2. Blood Adv 5:2569-2574. https://doi.org/10.1182/BLOODADVANCES. 2021004904

8. Al-Mayhni T, Saber S, Stubbs MJ et al (2021) Ischaemic stroke as a presenting feature of ChAdOx1 $\mathrm{nCoV}-19$ vaccine-induced immune thrombotic thrombocytopenia. J Neurol Neurosurg Psychiatry. https://doi.org/10.1136/JNNP-2021-326984

9. Sánchez Van Kammen M, Aguiar De Sousa D, Poli S et al (2021) Characteristics and outcomes of patients with cerebral venous sinus thrombosis in SARS-CoV-2 vaccine-induced immune thrombotic thrombocytopenia. JAMA Neurol 78:1314-1323. https://doi.org/10.1001/JAMANEUROL.2021.3619

10. Krzywicka K, Heldner MR, Sánchez van Kammen M et al (2021) Post-SARS-CoV-2-vaccination cerebral venous sinus thrombosis: an analysis of cases notified to the European Medicines Agency. Eur J Neurol 28:3656-3662. https://doi.org/10.1111/ENE.15029
11. De Michele M, Iacobucci M, Chistolini A et al (2021) Malignant cerebral infarction after ChAdOx 1 nCov-19 vaccination: a catastrophic variant of vaccine-induced immune thrombotic thrombocytopenia. Nat Commun 12:4663. https://doi.org/10.1038/ S41467-021-25010-X

12. Walter U, Fuchs M, Grossmann A et al (2021) Adenovirus-vectored COVID-19 vaccine-induced immune thrombosis of carotid artery: a case report. Neurology. https://doi.org/10.1212/WNL. 0000000000012576

13. Kenda J, Lovrič D, Škerget M, Milivojević N (2021) Treatment of ChAdOx1 nCoV-19 vaccine-induced immune thrombotic thrombocytopenia related acute ischemic stroke J Stroke Cerebrovasc Dis 30.https://doi.org/10.1016/J.JSTROKECEREBROVASDIS. 2021.106072

14. Lioudaki S, Kontopodis N, Pontikoglou C et al (2021) Multiple sites of arterial thrombosis in a 35-year old patient after ChAdOx1 (AstraZeneca) vaccination, requiring emergent femoral and carotid surgical thrombectomy. Ann Vasc Surg. https://doi.org/ 10.1016/J.AVSG.2021.07.033

Publisher's note Springer Nature remains neutral with regard to jurisdictional claims in published maps and institutional affiliations. 